for blocking IL-6 in this disease mainly lays on the pro-inflammatory role of this cytokine in the disease. However, only few works have studied the consequences of anti-IL-6R treatment on Tregs cells and mainly focuses on their frequency. Our objective was to elucidate anti-IL-6R mode of action on Tregs in RA patients treated with tocilizumab and in a RA model.

Methods Mice with collagen-induced arthritis (CIA) were treated at day 0 by MR16-1 (a rat anti-mouse IL- 6 receptor monoclonal Ab provided by Chugai Pharmaceutical Co. LTD, Japan) and the evolution of $\mathrm{CD}^{+}{ }^{+} \mathrm{FoxP}^{+}$Tregs during arthritis course was assessed at key time points (day 8-18-28 and 42 after CIA induction) by studying their number, frequency and phenotype (expression of GITR, ICOS, Helios, CD62L, CTLA-4 and CD39) in lymph nodes (LN), thymus and spleen by flow cytometry. Numerical analysis of Th17 and Th1 cells was also performed by flow cytometry. Twenty patients with severe and active RA were recruited and treated with $8 \mathrm{mg} / \mathrm{kg}$ of tocilizumab monthly. Peripheral blood was recovered at day 0 , as well as 1 and 3 month, and Th17and Tregs were analysed by flow cytometry.

Results Clinical and histological evaluation of arthritides in mice treated with anti-mouse IL-6R mAb showed, as expected, a less severe disease as compared to control Ig treated mice. Th17 frequency was unchanged, but Tregs frequency was enhanced in the LN of MR16-1 treated mice. In the thymus, we observed an enhanced frequency of Tregs $\mathrm{CD}^{+}{ }^{+} \mathrm{CD} 8-\mathrm{FoxP} 3^{+}$. Tregs phenotype was also modified in treated mice, with an increased frequency of CD39+ Tregs (LN and spleen), suggesting an enhanced ATP hydrolysis immunosuppressive activity of Tregs. In RA patients, Th17 frequencies were not modified by tocilizumab therapy and did not differ between responders and non-responders. Interestingly, CD39+ Treg cell among CD4 ${ }^{+}$ cells frequencies were significantly higher in responders than in nonresponders after 3 months of tocilizumab therapy.

Conclusions Tregs, but not Th17, are modified by anti-IL-6R treatment in both CIA and RA. These results support a beneficial effect in RA of treatments responsible for CD39+ Tregs enhancement and emphasise the relevance of the monitoring cell populations after cytokine blockade used to treat arthritis.

\section{A3.11 IMMUNOSUPPRESSIVE EFFECTS OF GLUCOCORTICOIDS AND REGULATORY T CELLS ON CD28NULL T CELLS IN VITRO}

doi:10.1136/annrheumdis-2013-203216.11

Jayesh Pandya, Mohammad Shahadat Hossain, Ingrid E Lundberg, Vivianne Malmström. Rheumatology Unit, Department of Medicine, Karolinska University Hospital, Solna, Karolinska Institutet, Stockholm, Sweden

Background and Objectives CD28null T cells are terminally differentiated T cells lacking CD28 co-receptor. These cells display properties of proinflammatory killer cells and are suggested to be resistant to apoptosis in vivo. Frequencies of CD28null $\mathrm{T}$ cells are increased in various chronic, inflammatory diseases. CD28null $T$ cells dominate both in the affected muscle and peripheral blood of patients with idiopathic inflammatory myopathies (myositis), suggesting a role these cells in disease mechanism and muscle pathology. Recently, it was found in our lab that after conventional glucocorticoid treatment, the relative number of regulatory $T$ cells (Tregs) was unchanged or decreased, while the CD28null T cell proportion was mainly increased in muscle tissue of myositis patients. This lead to our working hypothesis that CD28null $\mathrm{T}$ cells are resistant to immunosuppression mediated by glucocorticoids in the setting of myositis. Such resistance could also be against Tregs mediated immunosuppression due to distinct phenotype of CD28null T cells. The aim of this study was to evaluate the immunosuppressive effects of glucocorticoids and Tregs on CD28null T cells in an in vitro system.
Method Peripheral blood mononuclear cells (PBMCs) were obtained from 3 healthy individuals using Ficoll separation. CD3+CD4+ $\mathrm{CD} 25++$ (high) cells were sorted as Tregs by flow cytometry. For glucocorticoid or Tregs mediated T cell suppression assays, PBMCs were stimulated with plate bound $\alpha-\mathrm{CD} 3$ antibody in presence of $4 \mathrm{uM}$ glucocorticoid (methyl prednisolone sodium succinate) or optimal proportion of Tregs. Up-regulation of the early activation marker CD69 was measured by flow cytometry. Suppression was estimated based on \% reduction in CD69 mean fluorescent intensity compared to stimulated control cells.

Results CD4+CD28null T cells (median \% suppression: 40.1\%) displayed lower sensitivity towards glucocorticoid-mediated suppression compared to CD28+ counterparts (median: $54.7 \%$ ), seen in all individuals tested. Similarly, CD4+CD28null T cells (median: 17.5\%) were less sensitive towards Tregs mediated suppression compared to CD28+ counterparts (median: $34.4 \%$ ) in all individuals. No clear trend could be observed in CD8 compartment so far.

Conclusions Although, more individuals need to be tested, the above in vitro data support our in vivo findings that CD28null $T$ cells are relatively resistant to glucocorticoid and Tregs mediated immunosuppression. Lower sensitivity of CD28null $\mathrm{T}$ cells towards glucocorticoid and Tregs mediated suppression support their treatment resistance nature in myositis and a role in chronic inflammation and autoimmunity.

\section{A3.12 INTRARENAL FOXP3 + REGULATORY T CELLS EXPANSION AND DECREASED NUMBER OF INFILTRATING CD4+ T CELLS IN MURINE LUPUS BY IL-2 THERAPY}

doi:10.1136/annrheumdis-2013-203216.12

${ }^{1,2} \mathrm{~A}$ Rose, ${ }^{1,2} \mathrm{JY}$ Humrich, ${ }^{1,2} \mathrm{G}$ Riemekasten. ' $G e r m a n$ Rheumatism Research Center (DRFZ), a Leibniz Institute, Berlin, Germany; 'University Hospital Charité, Rheumatology and Clinical Immunology, Berlin, Germany

Background and Objectives Systemic lupus erythematosus (SLE) is an autoimmune disease characterised by an acquired IL-2 deficiency, which leads to a homeostatic imbalance between regulatory T cells (Treg) and effector T cells (Tcon). Humrich et al, (2010) demonstrated that the IL-2 deficiency in diseased $(\mathrm{NZB} \times \mathrm{NZW})$ F1 mice can be rebalanced in lymphoid organs using a treatment with recombinant IL-2 (IL-2) by promoting the homeostatic proliferation of regulatory $\mathrm{T}$ cells. The aim of this study was to investigate the impact of IL-2 therapy on intrarenal Foxp3 + Treg and kidney infiltrating $\mathrm{CD} 4+$ cells in $(\mathrm{NZB} \times \mathrm{NZW}) \mathrm{F} 1$ mouse model of lupus nephritis.

Materials and Methods $(\mathrm{NZB} \times \mathrm{NZW})$ F1 mice with active nephritis were treated with recombinant IL-2 either over a short period or for a total of 30 days. Absolute numbers, phenotype and proliferation of kidney infiltrating CD4 $+\mathrm{T}$ cells were determined by flow cytometry.

Results $(\mathrm{NZB} \times \mathrm{NZW})$ F1 mice treated over a short term with IL-2 showed an enhanced proliferation of Foxp3+ Treg and increased numbers and frequency of CD4+Foxp3+ Treg compared to untreated treated control mice. On the other hand, long term IL-2 treatment did not result in a persistent expansion of the intrarenal Foxp3+ Treg population. Nevertheless, total numbers of kidney infiltrating CD4 $+\mathrm{T}$ cells were diminished and the CD4 $+\mathrm{T}$ con showed reduced signs of cellular activation.

Conclusions Our data indicates that short term IL-2 treatment is able to expand the size of the intrarenal Treg pool. In contrast, long term IL-2 treatment decreases the numbers of kidney infiltrating $\mathrm{CD} 4+\mathrm{T}$ cells. These results may in part explain the delay of disease progression induced by treatment with IL-2 and underline the important role of intrarenal Treg for the suppression of kidney disease in lupus mice. These results also provide additional rationales for an IL-2 based immunotherapy of human disease. 